\title{
Predictores de dependencia en mayores de 65 años: una revisión sistemática
}

\section{Predictors of dependence among people older than 65 years: a systematic review}

\author{
Gloriannys Baez y Ana Rosa Delgado
}

Universidad de Salamanca, España

Disponible online 31 de diciembre de 2013

\begin{abstract}
El objetivo de este trabajo fue revisar sistemáticamente los instrumentos que se utilizan para estudiar la dependencia y la discapacidad en las personas mayores de 65 años y los predictores empleados en el análisis de la trayectoria de deterioro funcional en esta población. La búsqueda se realizó en la base de datos Scopus de donde se seleccionaron, utilizando categorías creadas ad hoc, 23 investigaciones de los 734 resúmenes disponibles. Los instrumentos y predictores se codificaron según el formato de presentación, el dominio de actividad y la medida empleada (discapacidad o dependencia). Los resultados muestran limitaciones en la conceptualización y elección de las medidas, debido a que no se emplea una definición estandarizada de los constructos y a las interpretaciones inadecuadas de las puntuaciones de los tests. Investigaciones futuras deberían diferenciar explícitamente la dependencia de la discapacidad y validar adecuadamente los instrumentos ya existentes, o bien construir nuevos tests haciendo uso de modelos psicométricos modernos.
\end{abstract}

Palabras clave: Demencia, Dependencia, Discapacidad; Deterioro Funcional; Envejecimiento; Predictores; Factores de Riesgo.

This paper systematically reviews the tools used to study dependence and disability in individuals older than 65 years and the predictors employed in analysing the trajectory of functional decline in this population. The search was conducted using the Scopus database and 23 out of the 734 available research abstracts were selected using ad hoc categories. The instruments and predictors were coded according to display format, domain of activity, and outcome measurement (disability or dependence). The results show limitations in the way the measures are conceptualized and selected, due to the lack of a standardized definition of the concepts and the inappropriate interpretation of test scores. Future research should explicitly distinguish dependence from disability, and correctly validate existing instruments or construct new ones using modern psychometric models.

Keywords: Aging; Dementia; Dependency; Disability; Functional Impairment; Predictors; Risk Factors.

Correspondencia: Gloriannys Báez, Departamento de Psicología Básica, Psicobiología y Metodología. Avda. de la Merced 109-131. 37005 Salamanca. España. Email: Gloriannys@gmail.com. E-mail de la co-autora: adelgado@usal.es. 
La esperanza de vida en España ya supera los 82 años y va en aumento, lo cual no es equivalente a una vida sana e independiente. En España, por cada dos personas en activo, hay una persona, mayor de 6 años, dependiente (INE-INEBASE, 2013). Los mayores de 65 años conforman dos terceras partes de la población dependiente en España (INE-INEBASE, 2013), que tiene una tasa de dependencia de población envejecida de 25.8 (Eurostat, 2013). Además, el 31.17\% de los españoles con edades comprendidas entre los 65 y los 74 años necesita ayuda en sus actividades de la vida diaria (AVD), porcentaje que aumenta según la edad de los sujetos: es el $47.74 \%$ entre los 75 y los 84 años y el $64.55 \%$ en mayores de 85 años (INEINEBASE, 2013).

Es necesario tratar el tema de la discapacidad antes de hablar de dependencia ya que, aunque son conceptos distintos, están íntimamente relacionados. La discapacidad y el deterioro funcional juegan un papel importante en la independencia del individuo, papel que se refleja en sus necesidades de cuidado y asistencia y en el uso de tecnologías de apoyo en el hogar (Evans, 1984; IMSERSO, 2005; Verbrugge y Jette, 1994), lo cual no es razón para que se definan o midan con el mismo patrón que la dependencia.

La definición de discapacidad involucra las limitaciones en la funcionalidad física y/o psicológica en un contexto social determinado en comparación con lo esperado para su edad, sexo y situación social (Nagi, 1964; OMS, 2001). En la vejez, la discapacidad suele medirse mediante tareas, especialmente las de vida diaria básicas y/o instrumentales (Agüero-Torres, Hillerås, y Winblad, 2001; Verbrugge y Jette, 1994). Sin embargo, se debería abarcar otras áreas como las actividades propias de su rol social y/o familiar, de participación social y recreativas (Evans, 1984; IMSERSO, 2005; Nagi, 1964; OMS, 2001).

En cuanto a la dependencia, dado un nivel concreto de discapacidad, se define como la necesidad del sujeto de asistencia externa para realizar las tareas antes mencionadas (Verbrugge y Jette, 1994), sea mediante asistencia directa o indirecta, supervisión o uso de productos de apoyo. La OMS (2001), aunque no utiliza el término de dependencia en la Clasificación Internacional del Funcionamiento (CIF), la Discapacidad y la Salud, sí se refiere a la severidad dentro del dominio denominado "actividades y participación" mediante los conceptos de desempeño y capacidad: en ambos se puede incluir la asistencia de terceros o de productos y tecnologías de apoyo para tener una idea del grado de limitación en el funcionamiento con estas ayudas y sin ellas.

La demencia tiene un peso importante en las tasas de dependencia funcional en la población envejecida. Por sí misma implica un deterioro en las funciones cognitivas superiores y en la capacidad del sujeto de realizar tareas de la vida diaria. Este síndrome, que afecta a más de 35 millones de personas en el mundo, es la principal causa de institucionalización en el anciano, además de ser un gasto importante en el presupuesto de salud de los países europeos (OMS, 2012).
Esta revisión está inspirada en una cuestión que surge en la práctica profesional y que consideramos de interés no solo para los clínicos, sino también para todos los que participan en el cuidado de personas mayores. Realizar proyecciones de las trayectorias de dependencia funcional ayudaría a evaluar las consecuencias de la misma, incluyendo los gastos de personal y económicos esperados. Estos estudios suelen tener un carácter gubernamental y parten de hipótesis demográficas como las de diferimiento de la morbilidad y expansión de la morbilidad entre otras (Prieto, 2010). En España, se utilizan para este fin los datos de la Encuesta de Discapacidad, Autonomía Personal y Situación de Dependencia (INE-INEBASE, 2013) donde se incluyen distintas áreas de Actividades y Participación. Sin embargo, estas predicciones no pueden ser trasladadas a una persona concreta, sino que se refieren a patrones poblacionales. Cuando se trata de individuos ¿qué indicadores podemos utilizar para estimar el grado de futura dependencia? El estudio de la trayectoria de dependencia funcional en el anciano solo es posible si se cuenta con medidas adecuadas, razón por la que es relevante estudiar los instrumentos que se están aplicando actualmente. De ahí que el objetivo de este trabajo sea revisar sistemáticamente los instrumentos que se utilizan para estudiar la dependencia y la discapacidad en las personas mayores de 65 años, identificando los que se emplean para predecir el deterioro funcional. Los resultados permitirán señalar qué funciones cognitivas podrían servir como marcadores de dependencia en estados temprano de deterioro cognitivo y/o de síndromes demenciales.

\section{Materiales}

\section{Método}

En la primera fase de la revisión, se trabajó con 734 resúmenes seleccionados de Scopus con las palabras clave de interés para nuestro objetivo. En una segunda fase, se revisaron 23 artículos seleccionados mediante el análisis de los citados resúmenes.

\section{Procedimiento}

La búsqueda se llevó a cabo los días 14 y 15 de marzo del 2013 en Scopus. Las palabras clave fueron: aging, dependency, disability y dementia, escogidas del Tesauro de la Asociación Americana de Psicología (APA, 2007). Se realizaron dos combinaciones: (a) aging, dementia, dependency y (b) aging, dementia, disability. Al hacer la búsqueda no se filtró por fecha ni tipo de publicación y tampoco se utilizaron los límites disponibles en Scopus; solo se utilizó el operador booleano "AND".

Los 23 artículos revisados en la segunda fase fueron seleccionados tras (a) eliminar de los 881 resultados todos los duplicados, editoriales, cartas al editor y cualquier otro que no fuera un estudio publicado en una revista o que no tuviese un resumen disponible en Scopus; (b) codificar en categorías mutuamente exhaustivas y excluyentes creadas ad hoc los 734 resúmenes resultantes, incluyendo solamente los que estudiaran 
la discapacidad, la dependencia y sus posibles predictores. El proceso de categorización, selección y eliminación de los 881 resultados se encuentra resumido en la Figura 1.

Para la codificación de los resúmenes, se tomaron en consideración distintas características de la muestra: edad $(\geq 65$ años, $\leq 65$ años y no especificada), tipo (población normal, con demencia, discapacidad intelectual, patologías somáticas) y situación (institucionalizadas o no). Las investigaciones se clasificaron por el idioma de publicación, el tipo de revista, objetos de estudio, diseño, instrumentos y la dimensión de discapacidad/dependencia que intentaban medir según los criterios de la CIF. Con respecto a los instrumentos, se realizaron codificaciones según el formato de presentación, el dominio de la CIF medido, las medidas de resultado y las actividades de la vida diaria (AVD) que utilizaba para medir funcionalidad.

\section{Figura 1}

Diagrama de flujo de codificación y toma de decisiones de los resúmenes en la primera fase

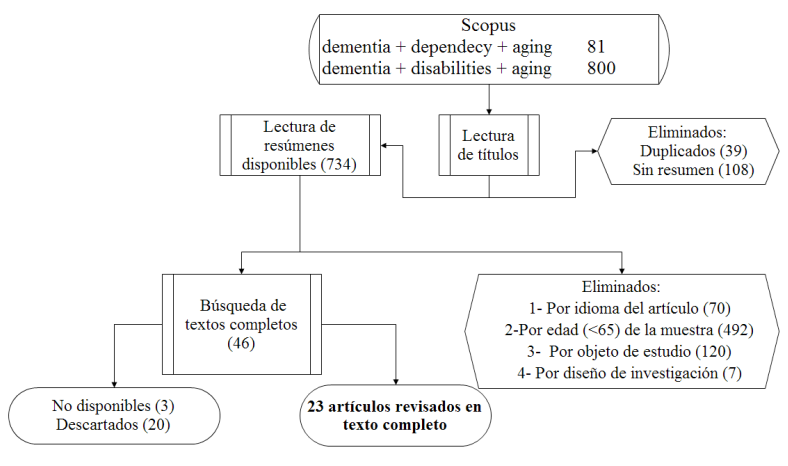

Haciendo uso de estas categorías se procedió a escoger los artículos que cumplieran los siguientes criterios: (a) publicados en español o inglés;(b) con muestras de edad igual o superior a 65 años; (c) cuyo objeto de estudio fueran los predictores de dependencia funcional y/o discapacidad. De los 46 artículos seleccionados para la segunda fase, solo tres no pudieron ser revisados. A pesar de que en la primera fase se habían excluido los resúmenes de investigaciones realizadas con muestras menores de 65 años, se halló que 13 artículos no cumplían realmente dicho requisito. Otros siete estudios fueron excluidos tras revisar los contenidos de las publicaciones, pues versaban sobre enfermedades y/o situaciones que, aunque relacionadas con la discapacidad (ej. caídas, roturas de cadera), no trataban sobre medidas de la misma. El corpus final de artículos revisados en la segunda fase consistió en 23 artículos completos que utilizaban 21 instrumentos y 124 posibles predictores.

\section{Análisis de datos}

El análisis de los resultados se llevó a cabo mediante un método mixto. Los resúmenes de la primera fase se codificaron mediante sistemas de categorías exhaustivas y mutuamente excluyentes creadas ad hoc (muestras, factores e instrumentos). Los instrumentos se codificaron según su formato, el dominio medido y las medidas empleadas.

\section{Resultados}

De todos los resúmenes disponibles, solo 11 provenían de publicaciones en español y 653 en inglés, un total de 664. En la Tabla 1 se presentan los resultados de la primera fase de la revisión (excluyendo los 70 resúmenes en otros idiomas) según la edad de la muestra, el diseño y su objeto de estudio.

La mayor parte de las investigaciones resumidas se codificaron en "no especificados" pues no estudiaban un grupo de edad determinado o bien se concentraban en los cuidadores, familiares y profesionales de salud involucrados en el tratamiento y asistencia de las personas mayores. Con respecto al diseño de los estudios, se clasificaron en observacionales, experimentales o revisiones. Una gran parte eran observacionales y revisiones, especialmente narrativas. Para una mejor lectura de la información en la Tabla 1, los estudios experimentales, revisiones y evaluaciones de instrumentos (que fueron tratados de forma diferente al inicio) se presentan bajo la designación de "otros".

Tabla 1

Frecuencia de Estudios por Edad, Diseño de Investigación y Objeto de Estudio

\begin{tabular}{|c|c|c|c|c|c|c|c|c|}
\hline & Epidemiología & Diagnóstico & $\begin{array}{l}\text { Cognición y/o } \\
\text { demencias }\end{array}$ & $\begin{array}{l}\text { Discapacidad/ } \\
\text { dependencia }\end{array}$ & $\begin{array}{l}\text { Morbilidad/ } \\
\text { mortalidad }\end{array}$ & Tratamiento & Otros & Total \\
\hline$\geq 65$ & 34 & 4 & 27 & 53 & 25 & 4 & 26 & 173 \\
\hline Obser $^{a}$ & 32 & 2 & 25 & 46 & 23 & 1 & 19 & 148 \\
\hline Otros & 2 & 2 & 2 & 7 & 2 & 3 & 7 & 25 \\
\hline$\leq 65$ & 13 & 12 & 13 & 11 & 6 & 11 & 33 & 99 \\
\hline Obser. & 11 & 10 & 10 & 9 & 5 & 7 & 20 & 72 \\
\hline Otros & 2 & 2 & 3 & 2 & 1 & 4 & 13 & 27 \\
\hline $\mathrm{N} / \mathrm{E}$ & 30 & 41 & 36 & 55 & 33 & 58 & 139 & 392 \\
\hline Obser. & 17 & 8 & 10 & 23 & 13 & 8 & 35 & 114 \\
\hline Otros & 13 & 33 & 26 & 32 & 20 & 50 & 104 & 278 \\
\hline Total & 77 & 57 & 76 & 119 & 64 & 73 & 198 & 664 \\
\hline
\end{tabular}

Nota: Total de resúmenes en castellano e inglés.

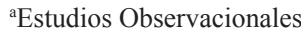


Un dato importante es la situación de la muestra: la mayoría vivían en comunidad y procedían de poblaciones no patológicas. Dentro de las patologías destaca la demencia, el deterioro cognitivo leve y los trastornos del neurodesarrollo, especialmente el síndrome de Down, considerados como envejecidos con respecto a su esperanza de vida.

Con respecto a la segunda fase de la revisión, la Tabla 2 muestra las principales características de los 23 artículos y los instrumentos utilizados. Gran parte de las investigaciones tienen un diseño transversal y muestras de población normal que viven en comunidad. De las 23 , solo una tiene como objeto de estudio la dependencia funcional, pues el resto, estrictamente hablando, se refiere a discapacidad. Los estudios han sido agrupados por el dominio de discapacidad que exploran según la CIF (OMS, 2001): veinte comprenden variables relacionadas con el dominio "actividades y participación"; un estudio trata el dominio "estructuras y funciones corporales"; y dos trabajan variables que pertenecen a ambos dominios.

Tabla 2

Resumen de los Artículos en Texto Completo Revisado

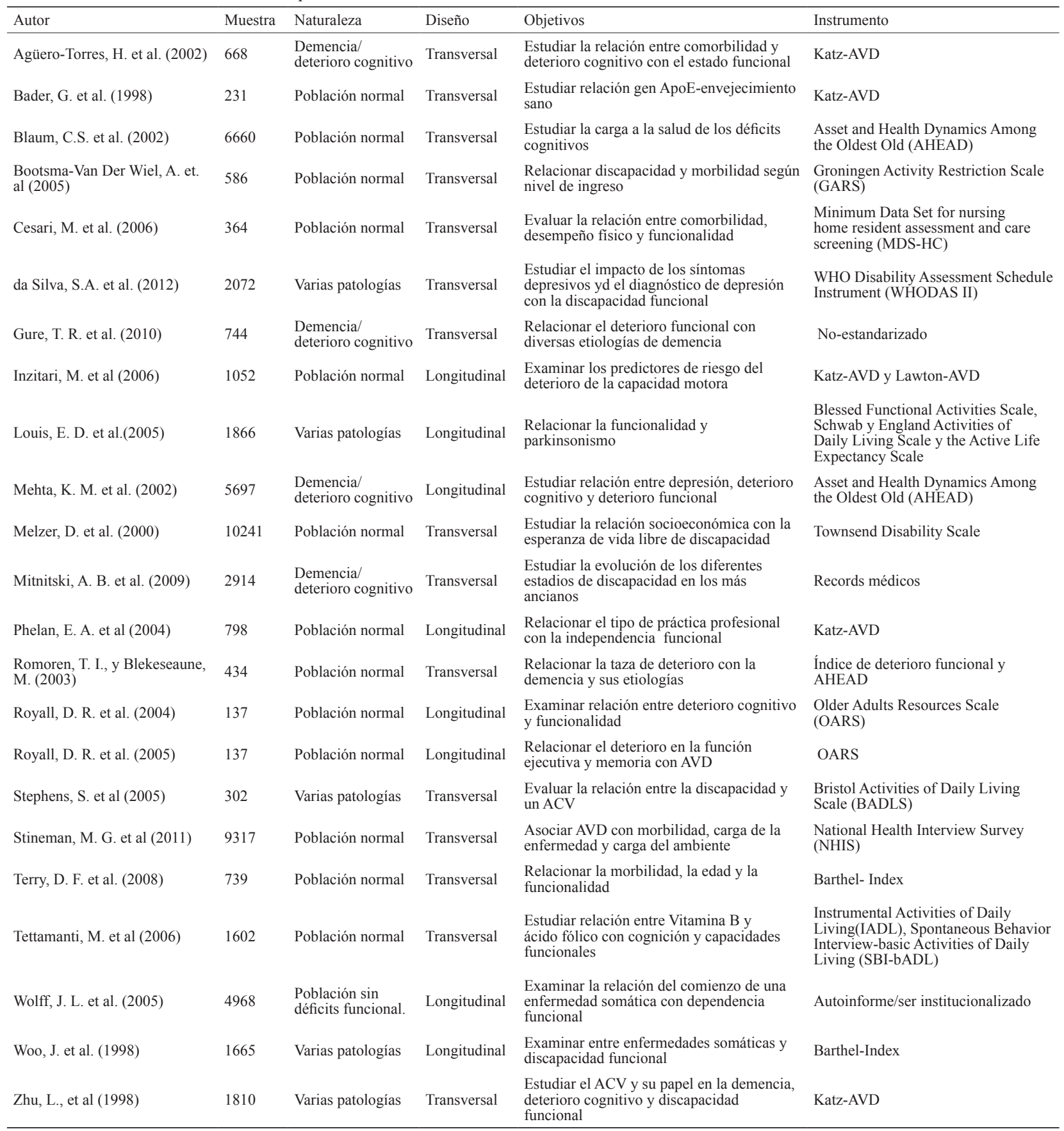


Se revisaron 21 medidas estandarizadas, de las cuales más de la mitad pretendían medir variables que corresponderían al dominio "actividades y participación" haciendo uso exclusivo de las AVD básicas e instrumentales. Los instrumentos más utilizados fueron el Katz Index of independence in activities of daily living y Barthel Index of Activities of Daily Living, ambos informes de capacidad para realizar AVD de manera independiente. Una descripción de cada medida estandarizada puede verse en la Tabla 3 según su formato de presentación, el dominio medido y la medida obtenida. Los tests se clasificaron a partir del artículo original o bien a partir de la versión empleada. Dos instrumentos, Active Life Expectancy Scale y Spontaneous Behavior Interview-basic Activities of Daily Living ( $S B I-b A D L)$, se codificaron utilizando la información suministrada por las investigaciones o la teoría en la que se sustentan. La codificación de dependencia o discapacidad en los instrumentos se llevó a cabo teniendo en cuenta los ítems utilizados, las escalas de respuestas y/o la normativa de corrección de la prueba (capaz/ incapaz, dependiente/ independiente).

Tabla 3

Instrumentos Según Dominio Medido, Formato de Presentación y Medida de Resultado

\begin{tabular}{|c|c|c|c|}
\hline Instrumentos & Dominio & Formato & Medida \\
\hline $4 \mathrm{~m}$ Test & Físicas & Desempeño & Discapacidad \\
\hline Active Life Expectancy Scale & AVD & Lápiz y papel & Discapacidad $^{\mathrm{a}}$ \\
\hline $\begin{array}{l}\text { Asset and Health Dynamics } \\
\text { Among the Oldest Old }\end{array}$ & AVD & Lápiz y papel & Discapacidad \\
\hline Barthel Index & AVD & Lápiz y papel & Dependencia \\
\hline $\begin{array}{l}\text { Blessed Functional Activities } \\
\text { Scale }\end{array}$ & AVD & Lápiz y papel & Discapacidad \\
\hline $\begin{array}{l}\text { Bristol Activities of Daily Living } \\
\text { Scale }\end{array}$ & AVD & Lápiz y papel & Dependencia \\
\hline $\begin{array}{l}\text { Canadian Study of Health and } \\
\text { Aging }\end{array}$ & Mixta & Lápiz y papel & Dependencia \\
\hline Fuerza Muscular & Físicas & Desempeño & Discapacidad \\
\hline $\begin{array}{l}\text { Groningen Activity Restriction } \\
\text { Scale }\end{array}$ & AVD & Lápiz y papel & Dependencia $^{\mathrm{b}}$ \\
\hline Katz-AVD & AVD & Lápiz y papel & Dependencia \\
\hline Lawton-AVD & AVD & Lápiz y papel & Dependencia \\
\hline Minimum Data Set & Mixta & Lápiz y papel & Dependencia \\
\hline MMSE & $\mathrm{n} / \mathrm{a}$ & Lápiz y papel & $\mathrm{n} / \mathrm{a}$ \\
\hline National Health Interview Survey & Mixta & Lápiz y papel & Discapacidad \\
\hline Neuromuscular Test & Físicas & Desempeño & Discapacidad \\
\hline Older Adults Resources Scale & Mixta & Lápiz y papel & Discapacidad \\
\hline $\begin{array}{l}\text { Schwab y England Activities of } \\
\text { Daily Living Scale }\end{array}$ & AVD & Lápiz y papel & Dependencia \\
\hline $\begin{array}{l}\text { Spontaneous Behavior Interview- } \\
\text { basic Activities of Daily Living }\end{array}$ & AVD & Lápiz y papel & Dependencia $^{c}$ \\
\hline $\begin{array}{l}\text { The Short Physical Performance } \\
\text { Battery }\end{array}$ & Físicas & Desempeño & Discapacidad \\
\hline Townsend Disability Scale & AVD & Lápiz y papel & Discapacidad \\
\hline $\begin{array}{l}\text { WHO Disability Assessment } \\
\text { Schedule Instrument }\end{array}$ & AVD & Lápiz y papel & Discapacidad \\
\hline
\end{tabular}

Nota: n/a no aplica.

aSegún la teoría en que se basa.

${ }^{b}$ Existe discordancia entre los ítems y la escala de respuesta.

'Según la investigación que lo utiliza.
Todas las pruebas revisadas que midieron el dominio "actividades y participación” y las mixtas están en formato de lápiz y papel, mientras que todas las de "estructuras y funciones" correspondían a tests de desempeño. De los 11 tests que miden AVD, cinco tenían medidas de discapacidad y los restantes de dependencia.

El Minimental State Examination (MMSE) fue utilizado como medida de funcionalidad en uno de los artículos revisados donde se consideró que aquellos sujetos con una puntuación menor o igual a 10 podían clasificarse como "necesitados de cuidado constante" sin importar su nivel de funcionalidad física y el desempeño en otras pruebas. Otro caso especial fue el Groningen Activity Restriction Scale debido a que, aunque sus ítems reflejan discapacidad, la escala de respuesta incluye una mezcla de los conceptos de discapacidad y dependencia.

En relación a los predictores, se encontraron 78 variables y 124 predictores de discapacidad/dependencia. De estos, 104 son simples y 20 combinados, es decir, estaban compuestos por múltiples variables y/o por dos medidas de discapacidad. Los predictores simples corresponden tanto a variables de sujeto como ajenas al mismo. Las variables de sujeto son: (a) medidas sociodemográficas como la edad, el sexo y nivel de ingresos; (b) biofisiológicas, relacionadas con estructuras corporales y sus patologías; (c) psicológicas, relacionadas con la cognición, factores emocionales y trastornos psiquiátricos. Por su parte, los ajenos al sujeto se refieren a la experiencia de los profesionales de la salud ligados al tratamiento, el nivel de asistencia médica necesaria o el tipo de enfoque de intervención.

Los predictores simples están representados en la Tabla 4 según el tipo de variables utilizadas. Los más empleados fueron los biofisiológicos y los psicológicos. Los principales predictores biofisiológicos fueron los problemas cardiopulmonares y vasculares, seguidos de los relacionados con la estructura ósea y las articulaciones. Entre los predictores psicológicos destacan los de deterioro cognitivo leve o moderado y los de demencias clasificados por severidad y etiología. En cuanto a los dominios de la CIF, en "actividades y participación" es donde se concentran la mayor parte de los predictores, especialmente en las AVD.

Tabla 4

Frecuencias de Predictores Simples por Variables y Dominio Medido

\begin{tabular}{lccc}
\hline \multirow{2}{*}{ Predictores } & \multicolumn{2}{c}{$\begin{array}{c}\text { Dominios de discapacidad } \\
\text { dependencia }\end{array}$} & Total \\
\cline { 2 - 3 } & $\begin{array}{c}\text { Funciones y } \\
\text { estructuras }\end{array}$ & $\begin{array}{c}\text { Actividades y } \\
\text { participación }\end{array}$ & \\
\hline Sociodemográficas & 3 & 6 & 9 \\
Psicológicos & 5 & 23 & 28 \\
Biofisiológicos & 25 & 39 & 64 \\
Ajenos al sujeto & 0 & 3 & 3 \\
Total & 33 & 71 & 104 \\
\hline
\end{tabular}

Los predictores combinados se utilizaron por tres de las investigaciones. Phelan, Williams, LaCroix, Grothaus, LoGerfo y Wagner, (2004) e Inzitari et al. (2006) utilizaron 
una variable perteneciente a uno de los dominios de la CIF empleado para predecir variables que pertenecen al otro dominio. Mientras que Mitnitski, Graham, Mogilner y Rockwood (1999) agruparon los síntomas clínicos característicos del diagnóstico de demencia en un índice de deterioro funcional, que definieron como el promedio de la probabilidad estimada de que uno de los 26 signos estudiados se encuentre presente en un sujeto determinado. Este índice está conformado tanto por signos que abarcan medidas funcionales, cognitivas, emocionales y comportamentales.

\section{Discusión}

El objetivo principal de esta revisión sistemática era revisar los instrumentos que se emplean en el estudio de la dependencia y la discapacidad en sujetos mayores de 65 años, para con ello identificar los que se emplean como predictores de deterioro funcional. El análisis se realizó en dos fases: los resultados de la primera fase muestran que los estudios sobre dependencia son muy escasos en relación con otros temas como la morbilidad, mortalidad o el deterioro cognitivo. En torno a una quinta parte del total de los resúmenes correspondieron a investigaciones de discapacidad/dependencia y una mínima parte de estos eran exclusivamente de dependencia. La baja proporción de artículos revisados en texto completo con relación al total de resúmenes disponibles se vio influenciado por la edad de las muestras empleadas, un $67 \%$ de los resúmenes trabajaban con muestras menores de 65 años y/o trabajaban con los cuidadores; y por la presencia de estudios que trabajan la tasa de dependencia económica y la dependencia a sustancia psicoactivas. Destaca, además, el gran número de revisiones en comparación con los estudios observacionales y experimentales, el uso de muestras no patológicas que viven en comunidad y estudios que involucran a cuidadores, familiares y profesionales de la salud.

Los resultados de la segunda fase muestran que, en la vejez, la discapacidad se estudia principalmente con pruebas de lápiz y papel para AVD básicas e instrumentales, sin relación con otras áreas funcionales de la vida como las ligadas a los roles o las relaciones sociales y la actividades recreativas. Resultados similares han aparecido en otras revisiones (Agüero-Torres, Hillerås y Winblad, 2001; Gold, 2012; Law, 1993; Law y Letts, 1989). No obstante, existe una amplia selección de pruebas de desempeño que pueden ser utilizadas para la evaluación de las AVD en el anciano (Gold, 2012; Moore, Palmer, Patterson y Jeste, 2007).

Los humanos son seres biopsicosociales y los ancianos no son una excepción a esta regla. Los teóricos de la discapacidad (Nagi, 1964; OMS, 2001; Verbrugge y Jette, 1994) han dedicado sus mayores esfuerzos a evitar que esta se considere como un concepto unidimensional, sobrepasando la concepción de enfermedad y concentrándose en las consecuencias en distintas áreas. El estudio de la discapacidad en el anciano debería llevarse a cabo teniendo en cuenta todas las áreas de actividades y participación, como en el resto de poblaciones, sin limitarse solo a AVD, y utilizando medidas de desempeño de alto valor ecológico, como el Texas Functional Living Scale (TFLS), el Direct Assesment of Functional Abilities (DAFA) u otras de las presentadas por Moore et al. (2007) en su revisión, para poder extraer conclusiones relativas a la prevención, recuperación y compensación del deterioro.

Un aspecto a destacar es el concepto mismo de discapacidad y dependencia utilizado explícita o implícitamente en las investigaciones. A pesar de los intentos de contar con un lenguaje común en esta área (OMS, 2001), muchas investigaciones trataron el tema de la discapacidad con definiciones e instrumentación correspondientes a lo que normativamente se entiende como dependencia. Por ejemplo, la mayoría de los tests estandarizados de AVD midieron, en realidad, dependencia cuando pretendían medir discapacidad. Este problema ha sido señalado por algunos autores como una amenaza a la validez de las interpretaciones de los resultados de dichas investigaciones (Tak, Kuiper, Chorus y Hopman-Rock, 2013; Verbrugge y Jette, 1994).

Un instrumento ve menguada su validez cuando no incluye ítems correspondientes a los diferentes dominios que se pretende medir, cuando las respuestas se ven influidas por variables extrañas o las interpretaciones ofrecidas no concuerdan con las teorías que lo fundamentan (Prieto y Delgado, 2010). La existencia de discordancia entre las medidas y las interpretaciones que se realizan a partir de ellas es uno de los principales resultados encontrados en esta revisión y constituye una importante limitación de las investigaciones en este campo. Los resultados de una prueba de discapacidad o dependencia tienen una gran repercusión en la vida de las personas, pues pueden afectar su autonomía, su libertad y sus responsabilidades por lo que deben ser interpretados teniendo en cuenta sus consecuencias.

A estas limitaciones metodológicas encontradas en los instrumentos, se une el hecho de que no se utilizan test basados en la Teoría de Respuesta al Ítem (TRI) que no presentan muchas de las debilidades que suelen tener los test que están basados en la Teoría Clásica de los Test (TCT) (Muñiz, 2010; Wright y Mok, 2004). Las pruebas utilizadas actualmente no contemplan la variabilidad de los ítems que las componen y por ello sitúan en el mismo nivel tareas complejas, instrumentales y básicas. Sin embargo, es cuestionable que la incapacidad de mantener el control de esfínteres se localice en el mismo nivel del constructo que las dificultades para hacer las tareas del hogar o cortarse las uñas de los pies. Una posible solución a este problema es el uso de modelos de medida avanzados, específicamente el modelo de Rasch (para detalles sobre el modelo ver Muñiz, 2010).

Aunque varios de los posibles predictores revisados tuvieron relación con las medidas de dependencia/discapacidad, los fallos en la conceptualización y en la selección de las pruebas psicométricas afectan a la validez e impiden la generalización de las interpretaciones. No obstante, cabe destacar el uso de las pruebas de demencia como predictores de la trayectoria del 
deterioro funcional en el anciano. La demencia es un síndrome y, como tal, está conformado por numerosos signos que fueron ignorados, en la selección de predictores, en una amplia proporción de las investigaciones. No se debería considerar la demencia como factor de riesgo de la dependencia, ya que forma parte de la definición: sin deterioro funcional, no es posible obtener el diagnostico de demencia (APA, 2000). Resulta más recomendable la utilización de signos cuantificables del síndrome, especialmente los de carácter cognitivo. Algunos déficits cognitivos en la memoria episódica, la atención y la fluencia verbal se han asociado con una disminución de la funcionalidad, incluso en poblaciones sin diagnóstico de demencia (Mariani, et al., 2008; Weston, Barton, Lesselyong y Yaffe, 2011), pero aún es pronto para determinar si podrían servir como marcadores de futura dependencia.

En conclusión, las limitaciones en la conceptualización de las medidas deberían ser tomadas en consideración partiendo de definiciones que diferencien claramente la dependencia de la discapacidad. También podrían someterse a prueba, con modelos psicométricos avanzados, los instrumentos existentes. Y, además, deberían construirse nuevos instrumentos de medida para el estudio de la dependencia, utilizando conceptos claros y que abarquen todas las áreas de actividades y participación. Es necesario contar con un lenguaje común que permita incrementar el conocimiento científico sobre el deterioro funcional, sus predictores y sus consecuencias.

\section{Referencias}

1. Agüero-Torres, H., Hillerås, P. y Winblad, B. (2001). Disability in activities of daily living among the elderly. Current Opinion in Psychiatry, 14, 355-359. http://dx.doi. org/10.1097/00001504-200107000-00020

2. Agüero-Torres, H., Thomas, V.S., Winblad, B. y Fratiglioni, L. (2002). The impact of somatic and cognitive disorders on the functional status of the elderly. Journal of Clinical Epidemiology, 55, 1007-1012. http://dx.doi.org/10.1016/ S0895-4356(02)00461-4

3. American Psychiatric Association. (2000). Diagnostic and Statistical Manual of Mental Disorders, Fourth Edition: DSM-IV-TR ${ }^{\circledR}$, Arlington: American Psychiatric Association.

4. American Psychological Association (2007). Thesaurus of psychological index terms. Washington, American Psychological Association.

5. Bader, G., Zuliani, G., Kostner, G.M. y Fellin, R. (1998). Apolipoprotein E polymorphism is not associated with longevity or disability in a sample of Italian octo- and nonagenarians. Gerontology, 44, 293-299. http://dx.doi. org/10.1159/000022030

6. Blaum, C.S., Ofstedal, M.B. y Liang, J. (2002). Low cognitive performance, comorbid disease, and task-specific disability: Findings from a nationally representative survey. Journals of Gerontology - Series A Biological Sciences and Medical Sciences, 57, M523-M531. http://dx.doi. org/10.1093/gerona/57.8.M523

7. Bootsma-Van Der Wiel, A., De Craen, A. J.M., Van Exel, E., Macfarlane, P. W., Gussekloo, J. y Westendorp, R.G.J. (2005). Association between chronic diseases and disability in elderly subjects with low and high income: The Leiden 85-plus Study. European Journal of Public Health, 15, 494-497. http://dx.doi.org/10.1093/eurpub/cki015

8. Cesari, M., Onder, G., Russo, A., Zamboni, V., Barillaro, C., Ferrucci, L., ... Landi, F. (2006). Comorbidity and physical function: Results from the aging and longevity study in the sirente geographic area (iISIRENTE Study). Gerontology, 52, 24-32. http://dx.doi.org/10.1159/000089822

9. Da Silva, S. A., Scazufca, M. y Menezes, P. R. (2013). Population impact of depression on functional disability in elderly: results from "São Paulo Ageing y Health Study" (SPAH). European archives of psychiatry and clinical neuroscience, 263, 153-158. http://dx.doi.org/10.1007/ s00406-012-0345-4

10. European Commision Statistics Database, Eurostats. (2013). Old Age Dependency ratio. Recuperado 16 de septiembre 2013 en http://epp.eurostat.ec.europa.eu/tgm/ table.do? tab $=$ tableyinit $=1$ yplugin $=1$ ylanguage $=$ enypcode $=$ $\underline{\text { tsdde } 510}$

11. Evans, J. G. (1984). Prevention of age-associated loss of autonomy: Epidemiological approaches. Journal of Chronic Diseases, 37, 353-363. http://dx.doi.org/10.1016/00219681(84)90101-2

12. Gold, D. A. (2012). An examination of instrumental activities of daily living assessment in older adults and mild cognitive impairment. Journal of clinical and experimental neuropsychology, 34, 11-34. http://dx.doi.org/ $10.1080 / 13803395.2011 .614598$

13. Gure, T. R., Kabeto, M. U., Plassman, B. L., Piette, J. D., y Langa, K. M. (2010). Differences in functional impairment across subtypes of dementia. Journals of Gerontology - Series A Biological Sciences and Medical Sciences, 65, 434-441. http://dx.doi.org/10.1093/gerona/glp197

14. IMSERSO (2011): Libro blanco del envejecimiento activo. Madrid. Disponible en: http://www. imserso.es/InterPresent1/groups/imserso/documents/ binario/8088 8089libroblancoenv.pdf

15. IMSERSO (2005): Libro blanco: Atención de las personas en situación de dependencia en España. Madrid. Disponible en: http://www.dependencia.imserso.es/dependencia 01/ documentacion/antecedentes/libro blanco/index.htm

16. INE-INEBASE (2013): Encuesta sobre Discapacidad, Autonomía Personal y Situaciones de Dependencia (EDAD). Madrid. Disponible en: http://www.dependencia. imserso.es/dependencia_01/tramitacion/valoracion_dependencia/inst/index.htm

17. Inzitari, M., Di Carlo, A., Baldereschi, M., Pracucci, G., Maggi, S., Gandolfo, C., Bonaiuto, S., Farchi, G., Scafatp, 
E., Carbonin, P. e Inzitari, D. (2006). Risk and predictors of motor-performance decline in a normally functioning population-based sample of elderly subjects: The Italian longitudinal study on aging. Journal of the American Geriatrics Society, 54, 318-324. http://dx.doi.org/10.1111/j.1532$\underline{5415.2005 .00584 . \mathrm{x}}$

18. Law, M. (1993). Evaluating activities of daily living: directions for the future. The American journal of occupational therapy: official publication of the American Occupational Therapy Association, 47, 233-237. http://dx.doi. org/10.5014/ajot.47.3.233

19. Law, M. y Letts, L. (1989). A critical review of scales of activities of daily living. The American journal of occupational therapy: official publication of the American Occupational Therapy Association, 43, 522-528. http://dx.doi. org/10.5014/ajot.43.8.522

20. Louis, E. D., Tang, M. X., Schupf, N. y Mayeux, R. (2005). Functional correlates and prevalence of mild parkinsonian signs in a community population of older people. Archives of Neurology, 62, 297-302. http://dx.doi.org/10.1001/archneur.62.2.297

21. Mariani, E.R., Monastero, R., Ercolani, S., Rinaldi, P., Mangialasche, R., Costanzi,E., Vitale, D. F., Senin U. y Mecocc, P. (2008). Influence of comorbidity and cognitive status on instrumental activities of daily living in amnestic mild cognitive impairment: results from the ReGAl project. International journal of geriatric psychiatry, 23, 523-530. http://dx.doi.org/10.1002/gps.1932

22. Mehta, K. M., Yaffe, K. y Covinsky, K. E. (2002). Cognitive impairment, depressive symptoms and functional decline in older people. Journal of the American Geriatrics Society, 50, 1045-1050. http://dx.doi.org/10.1046/j.15325415.2002.50259.x

23. Melzer, D., McWilliams, B., Brayne, C., Johnson, T. y Bond, J. (2000). Socioeconomic status and the expectation of disability in old age: Estimates for England. Journal of Epidemiology and Community Health, 54, 286-292. http:// dx.doi.org/10.1136/jech.54.4.286

24. Mitnitski, A. B., Graham, J. E., Mogilner, A. J. y Rockwood, K. (1999). The rate of decline in function in Alzheimer's disease and other dementias. Journals of Gerontology Series A Biological Sciences and Medical Sciences, 54, M65-M69. http://dx.doi.org/10.1093/gerona/54.2.M65

25. Moore, D. J., Palmer, B. W., Patterson, T. L. y Jeste, D. V. (2007). A review of performance-based measures of functional living skills. Journal of psychiatric research, 41, 97-118. http://dx.doi.org/10.1016/j.jpsychires.2005.10.008

26. Muñiz, J. (2010). Las teorías de los tests: Teoría clásica y teoría de respuesta a los ítems. Papeles del Psicólogo, 31, 57-66.

27. Nagi, S. Z. (1964). A Study in the Evaluation of Disability and Rehabilitation Potential. American Journal of Public Health and the Nations Health, 54, 1568-1579. http://
dx.doi.org/10.2105/AJPH.54.9.1568

28. Organización Mundial de la Salud (2001): Clasificación Internacional del Funcionamiento, de la Discapacidad y de la Salud (CIF): OMS y MTAS. Disponible en: http://www. imsersomayores.csic.es/documentos/documentos/oms-clasificacion-01.pdf

29. Organización Mundial de la Salud (2012): Dementia a public health priority. Disponible en: http://www.who. int/mental health/publications/dementia report 2012/en/ index.html

30. Phelan, E. A., Williams, B., LaCroix, A. Z., Grothaus, L., LoGerfo, J. P., y Wagner, E. H. (2004). Effects of provider practice on functional independence in older adults. Journal of the American Geriatrics Society, 52, 1233-1239. http://dx.doi.org/10.1111/j.1532-5415.2004.52350.x

31. Prieto, C. V. (2010). Estimación de la dependencia en España a partir de la EDAD 2008. Hacienda Pública Española, 194, 125-175.

32. Prieto, G., y Delgado, A. R. (2010). Fiabilidad y Validez. Papeles del Psicólogo, 31, 67-74.

33. Romoren, T. I., y Blekeseaune, M. (2003). Trajectories of disability among the oldest old. Journal of Aging and Health, 15, 548-566. http://dx.doi.org/10.1177/0898264303253633

34. Royall, D. R., Palmer, R., Chiodo, L. K. y Polk, M. J. (2004). Declining Executive Control in Normal Aging Predicts Change in Functional Status: The Freedom House Study. Journal of the American Geriatrics Society, 52, 346352. http://dx.doi.org/10.1111/j.1532-5415.2004.52104.x

35. Royall, D. R., Palmer, R., Chiodo, L. K. y Polk, M. J. (2005). Executive control mediates memory's association with change in instrumental activities of daily living: The freedom house study. Journal of the American Geriatrics Society, 53, 11-17. http://dx.doi.org/10.1111/j.15325415.2005.53004.x

36. Stephens, S., Kenny, R. A., Rowan, E., Kalaria, R. N., Bradbury, M., Pearce, R., Ballard, C. G. (2005). Association between mild vascular cognitive impairment and impaired activities of daily living in older stroke survivors without dementia. Journal of the American Geriatrics Society, 53, 103-107. http://dx.doi.org/10.1111/j.15325415.2005.53019.x

37. Stineman, M. G., Xie, D., Pan, Q., Kurichi, J. E., Saliba, D. y Streim, J. (2011). Activity of daily living staging, chronic health conditions, and perceived lack of home accessibility features for elderly people living in the community. Journal of the American Geriatrics Society, 59, 454-462. http:// dx.doi.org/10.1111/j.1532-5415.2010.03287.x

38. Tak, E., Kuiper, R., Chorus, A. y Hopman-Rock, M. (2013). Prevention of onset and progression of basic ADL disability by physical activity in community dwelling older adults: a meta-analysis. Ageing research reviews, 12, 329-338. http://dx.doi.org/10.1016/j.arr.2012.10.001

39. Terry, D. F., Sebastiani, P., Andersen, S. L. y Perls, T. T. 
(2008). Disentangling the roles of disability and morbidity in survival to exceptional old age. Archives of Internal Medicine, 168, 277-283. http://dx.doi.org/10.1001/ archinternmed.2007.75

40. Tettamanti, M., Garrì, M. T., Nobili, A., Riva, E. y Lucca, U. (2006). Low folate and the risk of cognitive and functional deficits in the very old: The Monzino 80-plus study. Journal of the American College of Nutrition, 25, 502-508. http://dx.doi.org/10.1080/07315724.2006.10719565

41. Verbrugge, L. M. y Jette, A. M. (1994). The disablement process. Social Science and Medicine (1982), 38, 1-14. http://dx.doi.org/10.1016/0277-9536(94)90294-1

42. Weston, A., Barton, C., Lesselyong, J. y Yaffe, K. (2011). Functional deficits among patients with mild cognitive impairment. Alzheimer's y Dementia, 7, 611-614. http:// dx.doi.org/10.1016/j.jalz.2010.12.011

43. Wolff, J. L., Boult, C., Boyd, C. y Anderson, G. (2005). Newly reported chronic conditions and onset of functional dependency. Journal of the American Geriatrics Society, 53, 851-855. http://dx.doi.org/10.1111/j.1532-5415.2005.5326
44. Woo, J., Ho, S. C., Yu, L. M., Lau, J. y Yuen, Y. K. (1998). Impact of chronic diseases on functional limitations in elderly Chinese aged 70 years and over: A cross-sectional and longitudinal survey. Journals of Gerontology - Series A Biological Sciences and Medical Sciences, 53, M102M106. http://dx.doi.org/10.1093/gerona/53A.2.M102

45. Wright, B. D. y Mok, M. M. C. (2004). An Overview of the Family of Rasch Measurement Models. En: E.V. Smith y R.M. Smith (Eds.), Introduction to Rasch Measurement (pp. 1-24). Maple Grove: Jam Press.

46. Zhu, L., Fratiglioni, L., Guo, Z., Agüero-Torres, H., Winblad, B. y Viitanen, M. (1998). Association of stroke with dementia, cognitive impairment, and functional disability in the very old: A population-based study. Stroke, 29, 2094 209. http://dx.doi.org/10.1161/01.STR.29.10.2094

Fecha de recepción: 29 de junio de 2013 Fecha de recepción de la versión modificada: 8 de octubre de 2013 Fecha de aceptación: 20 de octubre de 2013 